\title{
Charge determination for high-z nucleus by fine grain nuclear emulsion
}

\section{Osamu Sato*, T.Naka, M.Yoshimoto, S.Tada}

Nagoya University

E-mail: sato@flab.phys.nagoya-u.ac.jp

\section{A.Ariga, T.Ariga, M.Vladymyrov}

BERN University

The idea and a performance test for detection of highly ionizing particles such as magnetic monopole with fine grain nuclear emulsion is presented. The fine grain emulsion named Nano Imaging Tracker (NIT) developed for dark matter search is considered to use as Emulsion Cloud Chamber (ECC) with standard emulsion in such an experiment. NIT is less sensitive for minimum ionizing particles (MIP) and measure energy deposition by highly ionizing particles without counting $\delta$ rays. While MIP sensitive standard emulsion measure the particle velocity by $\delta$ rays activities. The particle velocity and corresponding charge $\mathrm{z}$ estimated by the energy deposition are expected to be measured with the ECC. A test exposure to $13 \mathrm{~A} \mathrm{GeV}$ and $150 \mathrm{~A} \mathrm{GeV}$ lead beam at CERN was carried out and the aimed performance was confirmed that only core of trajectory of lead ions are recorded in NIT. Thanks to very good spacial resolution, lead ion interaction products can be seen even two tracks separation within one $\mu \mathrm{m}$. The vertically exposed NIT films can be readout with recent high speed automated track selector. The results of the test exposure shows a large area $\mathscr{O}\left(1000 \mathrm{~m}^{2}\right)$ ECC detector for search of exotic particles having high energy deposition can be possible.

The 3rd International Symposium on "Quest for the Origin of Particles and the Universe" 5-7 January 2017

Nagoya University, Japan

\footnotetext{
* Speaker.
} 


\section{Introduction}

Thanks to continuous improvements of track readout speed of automatic microscope scanning systems (called track selector)[1][2], a nuclear emulsion detector having large surface area for searching exotic particles can be considered now. Some exotic particles are expected to make high energy deposition such as magnetic monopole. The trajectory could be recognized similar as high$\mathrm{z}$ nucleus track. While high-z particles emit huge number of $\delta$ rays and it makes the track radius large and difficult to see the track itself as shown in Figure 10 The magnetic monopole[3] [4] will be recognized by large energy depositing track. The expected energy deposition is same as high charged $(\mathrm{z} \sim 68.5 \times n)$ track at relativistic speed. So the existence of huge amount of $\delta$ rays, it will be recorded also as large radius track by standard nuclear emulsion. While at standard usage of automatic track selector, it is tuned for minimum ionizing particles (MIP) and large radius tracks are not detected efficiently.

An idea arises to solve the situation using less sensitivity nuclear emulsion to ignore $\delta$ rays and only core region of high-z track trajectory to be recorded for readout at standard usage of automatic track selector. The nuclear emulsion developed for dark matter experiment, Nano Imaging Tracker (NIT) [6] is a good candidate for tracking of hjgh-z particle or magnetic monopoles.

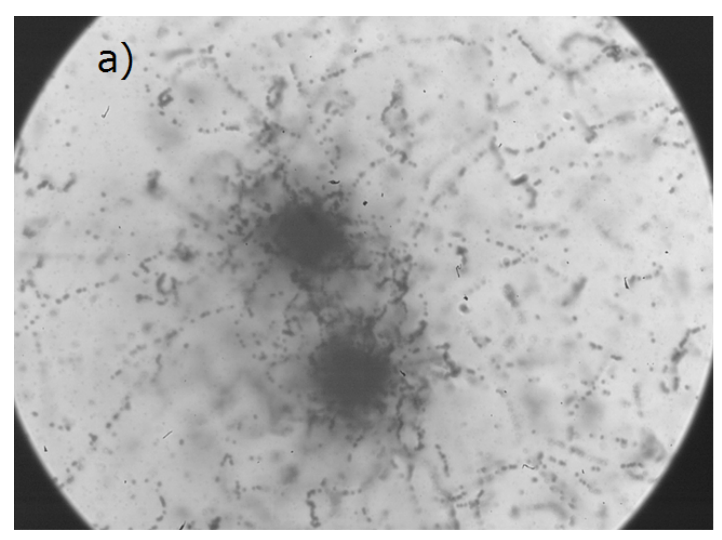

b)

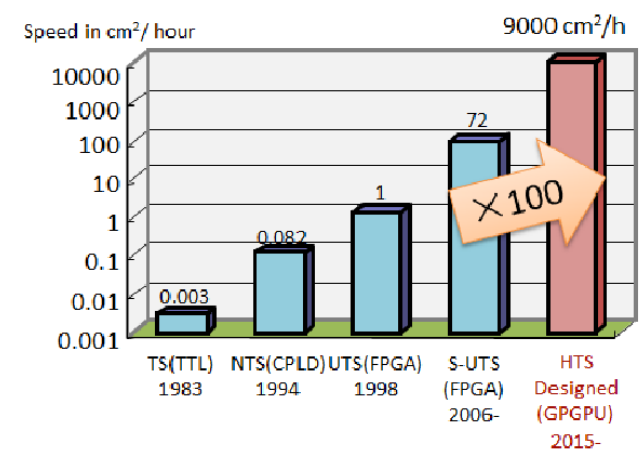

Figure 1: a) A microscope view of lead ions exposed to standard emulsion. Lead beam is perpendicular to this paper. b) Progress of automatic scanning speed(Japanese systems)

\section{Nano Imaging Tracker}

The nuclear emulsion is a detector having sub-micron spacial resolution and particle trajectories are recorded as three dimensional vectors. The nuclear emulsion made from $\mathrm{AgBr}$ crystals imerging in gelatin. The standard size of $\mathrm{AgBr}$ crystal is about $200 \mathrm{~nm}$. In 2010, an emulsion production facility are installed in Nagoya university F-lab. The tuning about AgBr crystal size is a main point for improving the nuclear emulsion feature. Nano Imaging Tracker (NIT) is developed in order to use for detection of dark matter signals. As shown in Figure2 the $\mathrm{AgBr}$ crystal size of NIT is $40 \mathrm{~nm}$ which allows to tracking very short range (oredr of $100 \mathrm{~nm}$ or less) scattered nucleus by WIMPs[6] [7]. 


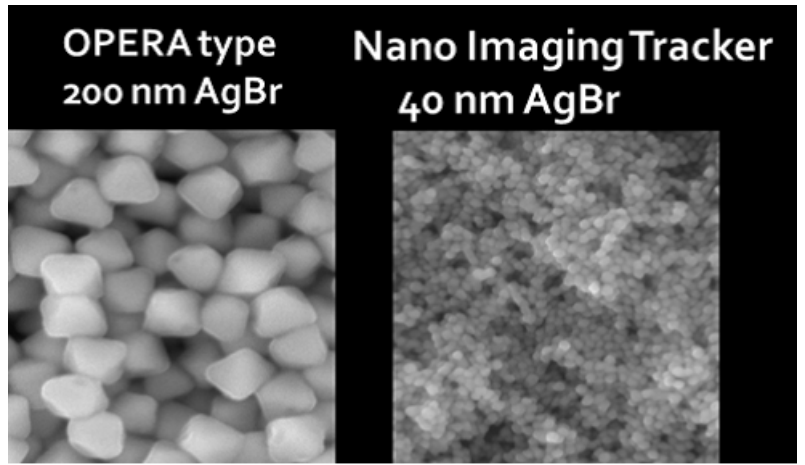

Figure 2: Electron microscope image of AgBr crystals, left OPERA type (200nm) and right NIT (40nm)

\section{Lead ion beam exposure test}

In order to know the NIT tracking performance, lead ion beam are exposure to Emulsion Cloud Chamber (ECC) stacked with NIT and standard nuclear emulsion (OPERA type [8). The test beam exposure were carried out at CERN H4 beam line at 18th November and 8th December 2016. As shown in Figure 3 two different type of ECC geometries were exposed. One is horizontally exposed and tracks can be seen from track's side direction in detail. Another is vertically exposed and tracks are recorded perpendicular to emulsion surface suit for readout by automatic track selectors. The ECCs were mounted on stage and $\mathrm{x}, \mathrm{y}$ position were controlled by target mover to accumulate lead ions uniformly in the ECC (Figure4).

a) horizontal exposure

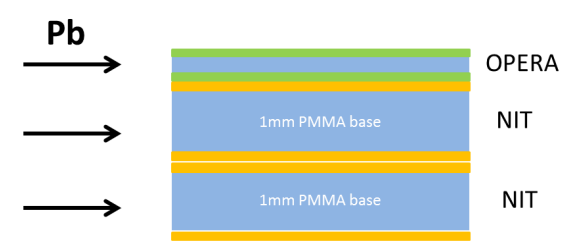

b) vertical exposure

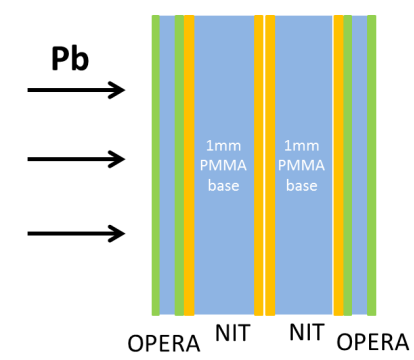

Figure 3: Shematic view of the ECC structure for a) horizontal and b) vertical exposure

After development of ECC films. The analysis of the exposed ECC have just been started. It turned out the idea was proven that NIT can recognize high-z particles as like MIP tracks recorded by OPERA type films. Almost all $\delta$ rays were not recorded in NIT but huge number of them are recorded in OPERA type films (Figure5). While the track radius (or thickness) were not a single value which is expected for pure lead ion beam $z=82$. It could be understood that the lead beam were contaminated with lead nucleus remnants by interactions with materials mainly about $3 \mathrm{~m}$ long air between beam ejected point to the ECC. The vertical exposed NIT films were scanned and beam tracks are recognized by standard use of track selector (Figure 6 ). So it open the possibility 

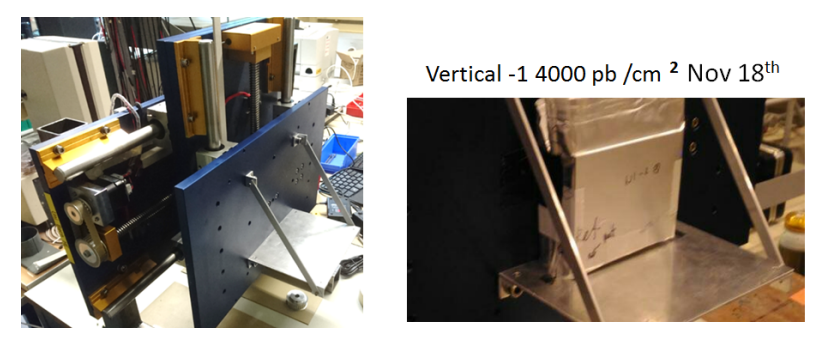

Figure 4: ECC mount table for the beam exposure: The ECCs are mounted on the table and the table are controlled to make accumulating track density uniform in whole ECC surface.

to perform a search for exotic particles having nature of high energy deposition with a large scale detector.
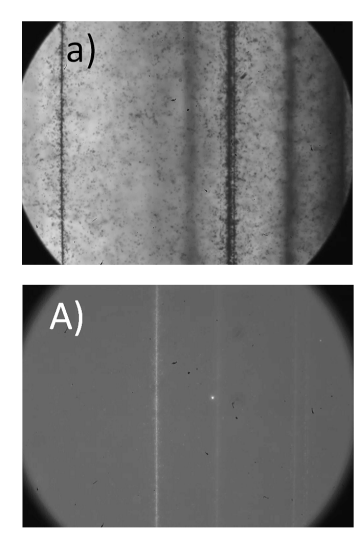
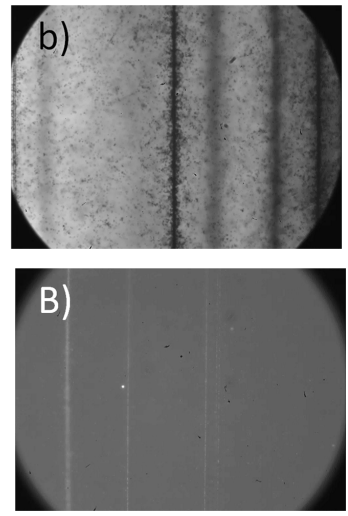
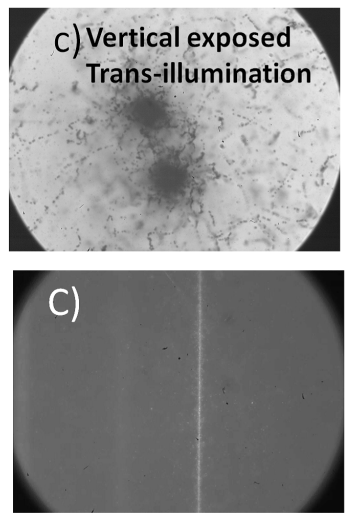
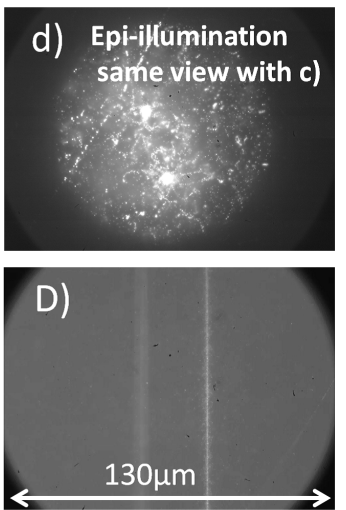

Figure 5: Microscope view of exposed films: upper OPERA type, bottom NIT films. c),d) is vertical exposed film and others are horizontal exposed film. Only c) is image view by Trans illumination, others are that of Epi-illumination. c) is the standard input for automatic track selector but track radius is too large to be recognized by standard usage. Track images in horizontal exposed film, a),b) and A),B),C),D) show different energy deposition along the beam tracks, they can be understood as broken parts of lead nucleus by interaction.

\section{Summary}

Fine grain emulsion, NIT have nice feature to detect high-z particles. It could be used as ECC with standard nuclear emulsion for charge determination of high-z particles and its velocity in magnetic monopole search. ECCs composed by NIT and OPERA type films were exposed to 13A $\mathrm{GeV}$ and 150A GeV lead ion beam in 2016 autumn. The analysis have just been started and first results are promising. At first, NIT have charge determination power for lead interacted remnants. And the tracks can be read out quickly by automatic scanning system with standard usage. A search for exotic particles having high energy deposition like magnetic monopole with a large surface area $\mathscr{O}\left(1000 \mathrm{~m}^{2}\right)$ detector of ECC become realistic. 
a) Track recognition pulse height \& pulse height volume

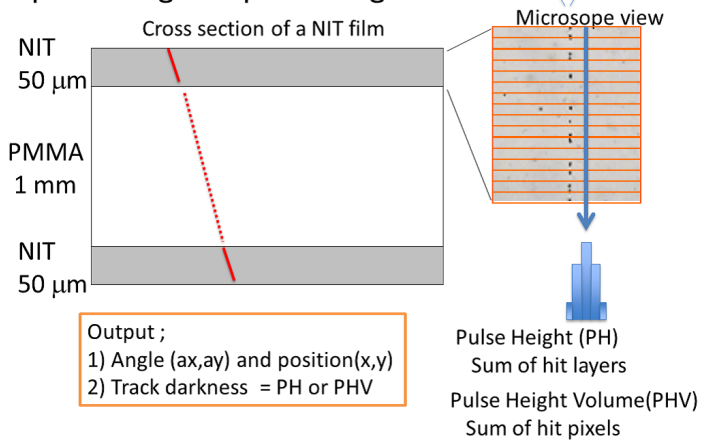

b) Scanning result pulse height \& pulse height volume

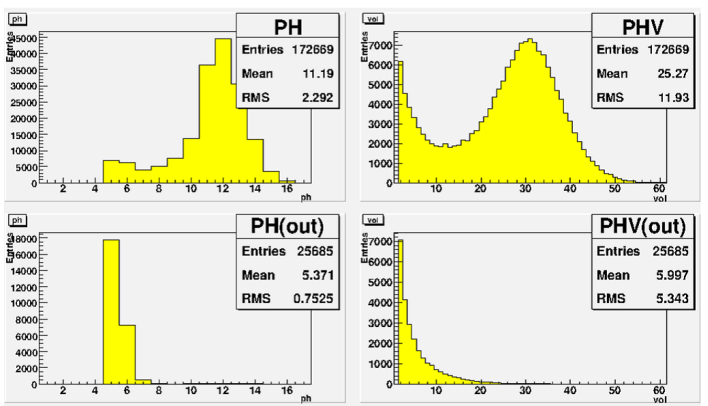

Figure 6: a) Track recognition algorithm on automatic track selector b) The Pulse Height (left )and Pulse Hight Volume (right) of scanned data of lead ion exposed film. Top part graph for track angle within 40mrad to beam angle and bottom is that of side band, ie. track angle more than 0.1 radian far from beam angle.

\section{References}

[1] Development of a new automatic nuclear emulsion system, K.Morishima et al. JINST 5 (2010) P04011

[2] An integrated system for large scale scanning of nuclear emulsions, C.Bozza et al., Nucl.Instrum.Meth .A703 (2013) 204-212

[3] Quantised Singularities in the Electromagnetic Field, P.A.Dirac, Proc.Roy.Soc.A 133(1931) 60

[4] Magnetic Monopoles in Unified Gauge theories, G.'t Hooft, Nucl.Phys.B79(1974) 276

[5] Evidence for Detector of a Moving Magnetic Monopole, P.B. Price et al., Phys.Rev.Lett. 35(8):487-490.

[6] Fine grained nuclear emulsion for higher resolution tracking detector, T.Naka et al., Nuck.Instrum.Meth.A718 (2013) 519-521

[7] New readout system for submicron tracks with nuclear emulsion, T.Katsuragawa et al., JINST 12(2017) NO.04, T04002

[8] The OPERA film: New nuclear emulsion for large-scale, high precision experiments, T.Nakamura et al. Nucl.Instrum.Meth.A556 (2006)80-86 